\title{
Immunization rates may not be so bad
}

$\mathrm{C}$ anada has a long way to go to achieve the same protection from vaccine-preventable diseases as other wealthy countries, however gaps in coverage may be smaller than previously estimated, said experts at the Canadian Immunization Conference on Dec. 2 in Ottawa.

Most developed nations maintain high levels of routine immunization, with rates averaging close to $95 \%$. However, among the 29 peer countries ranked in a 2013 UNICEF report, only Canada and Austria had rates lower than $85 \%$.

But in the absence of a national registry, or even complete provincial records, experts say that Canada's reported childhood immunization rate likely underestimates the actual number of children vaccinated.

"We have our fair share of data quality issues," explained Samara David, a surveillance epidemiologist at the $\mathrm{BC}$ Centre for Disease Control. Immunization registries are often incomplete, and may not include vaccinations provided outside public health - for example, by a physician or pharmacist. Because there are few links between registries, even within provinces, public health officials rely on parents to report which immunizations their children have received in other jurisdictions. Parents are also expected to report if they're moving away; when they don't, children have active, yet incomplete, immunization records across multiple registries.

Provinces are just beginning to get a handle how these problems skew reported immunization rates. $\mathrm{BC}$ previously estimated that about $64 \%$ of children born between 2004 and 2011 received appropriate immunization by age two. But when public health offi-

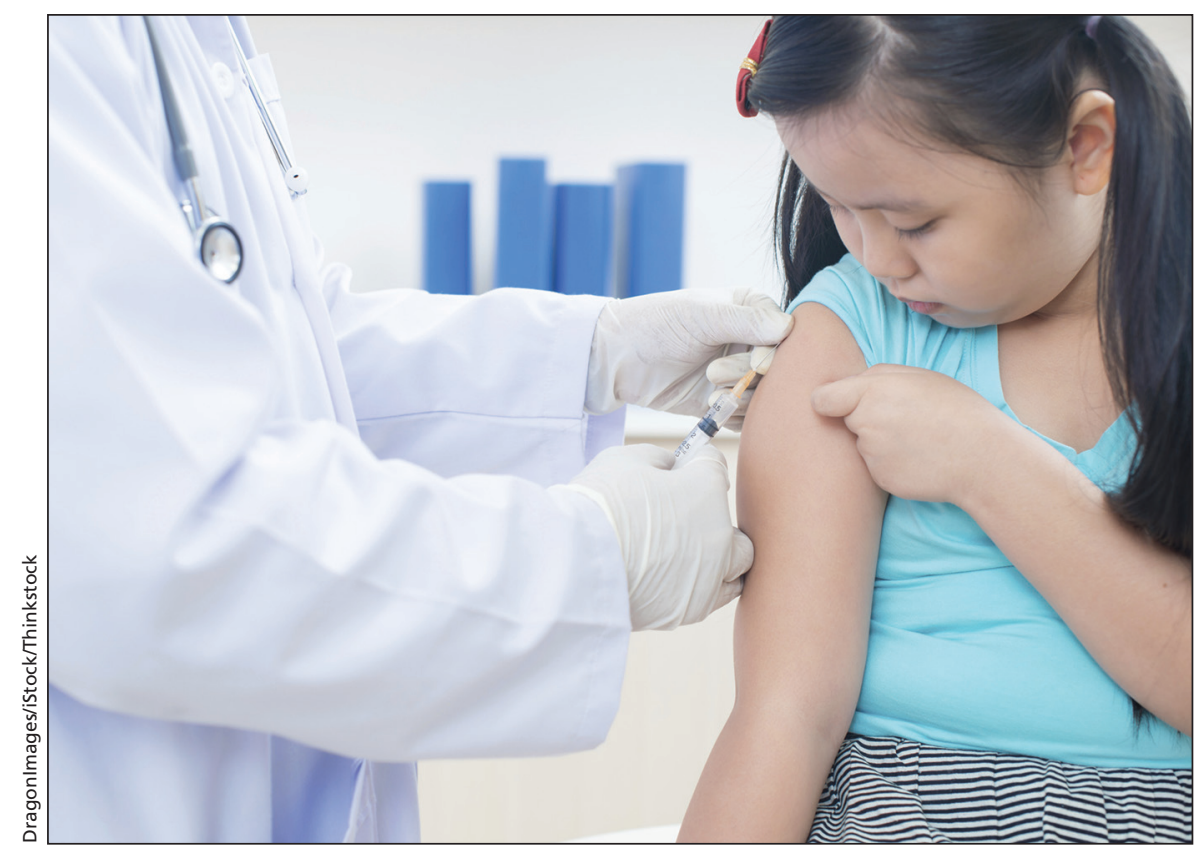

Gaps in immunization coverage may not be as extreme as previously thought, said presenters at the Canadian Immunization Conference in Ottawa.

cials followed up on a sample of children registered as "unimmunized" they discovered that $58 \%$ had been vaccinated for at least one disease and $28 \%$ were completely up to date.

"Based on these results, our revised estimates were that $71.5 \%$ of children were up to date, compared to $64 \%$ using the registry data alone, and only $3.1 \%$ of children were unimmunized, compared to $6 \%$ [an earlier estimate]," said David.

National telephone surveys of vaccination uptake fall prey to the same data quality issues. Coverage rates differ widely depending on whether survey participants have their immunization records in front of them during the call. They also differ when compared with health provider records, explained Julie Laroche, manager of Immuniza- tion Assessment and Information at the Public Health Agency of Canada. In some cases, "differences greater than $20 \%$ were found."

David said that there's an opportunity to improve the accuracy of immunization coverage estimates now that some provinces are making the long-awaited transition to run their registries on a shared system, called Panorama. She said other improvements might include providing registry access to immunizers outside of public health and possibly even parents, developing data linkages with electronic health records, and links with the Ministry of Health to automatically notify the immunization registry when a client moves. - Lauren Vogel, CMAJ

CMAJ 2015. DOI:10.1503/cmaj.109-4958 